\title{
Review of COVID-19 Vaccines and Their Evidence in Older Adults
}

\author{
Shyh Poh Teo \\ Department of Internal Medicine, Raja Isteri Pengiran Anak Saleha (RIPAS) Hospital, Brunei Darussalam
}

Corresponding Author:

Shyh Poh Teo, FRACP

Department of Internal Medicine, Raja

Isteri Pengiran Anak Saleha (RIPAS)

Hospital, Jalan Putera Al-Muhtadee

Billah, Bandar Seri Begawan, BA1710,

Brunei Darussalam

E-mail: shyhpoh.teo@moh.gov.bn

ORCID:

https://orcid.org/0000-0002-6117-5774

Received: January 19, 2021

Revised: February 4, 2021

Accepted: February 4, 2021
Coronavirus disease 2019 (COVID-19) caused by the severe acute respiratory syndrome coronavirus 2 (SARS-CoV-2) has resulted in a global pandemic and significant loss of life. Older people are vulnerable to SARS-CoV-2 infections and complications; thus, they are a priority group to receive COVID-19 vaccines. This review discusses considerations for COVID-19 vaccines for older adults. The general concepts of vaccine effectiveness in older adults are described, particularly immune senescence and vaccine development approaches to improve immunogenicity. The types of COVID-19 vaccine platforms are also described before reviewing the available, although limited, evidence from phase 3 COVID-19 vaccine trials relevant to older adults. The BNT162b2 vaccine by Pfizer-BioNTech and mRNA-1273 vaccine from Moderna demonstrated high efficacy and immunogenicity, which were also observed in older people. While the ChAdOx $1 \mathrm{nCoV}-19$ vaccine (AZD1222) by AstraZeneca demonstrated some efficacy in older people, the vaccine dose requires clarification through further studies. Finally, the Ad26.COV2.S vaccine by Janssen Pharmaceuticals shows promise as a single-dose vaccine with a potential durability of response.

Key Words: COVID-19, Aged, Older adults, Vaccines

\section{INTRODUCTION}

The infectious severe acute respiratory syndrome coronavirus 2 (SARS-CoV-2) has resulted in a global pandemic, causing significant disruption and loss of life. In response, there has been an unprecedented effort for the rapid development of vaccines to prevent coronavirus disease 2019 (COVID-19). As of January 1, 2021, 60 and 172 vaccines are in clinical and pre-clinical development, respectively. ${ }^{1)}$ The United States Food and Drug Administration has issued Emergency Use Authorizations approving the distribution and use of two vaccines in the United States: the BNT162b2 vaccine by Pfizer-BioNTech on December 11, 2020, and the mRNA-1273 vaccine from Moderna on December 18, 2020.

When COVID-19 vaccine programs are implemented, there will likely be limited supplies depending on the rates of vaccine production, shipping, and distribution and the ability to administer vaccines in mass-immunization programs. The World Health
Organization (WHO) Strategic Advisory Group of Experts offers guidance for prioritizing the allocation of available COVID-19 vaccines. People considered high-priority groups include essential workers to ensure continuity of critical services, people who are more likely to be exposed to and spread the virus, and finally, those with a higher risk of morbidity and mortality, such as older adults. ${ }^{3)}$

The present review discusses COVID-19 vaccine considerations for older adults. The general concept of vaccine effectiveness in older adults is described, followed by an overview of the types of COVID-19 vaccine platforms, before reviewing the currently available, although somewhat limited, evidence from phase 3 COVID-19 vaccine trials relevant to older adults.

\section{VACCINATION EFFECTIVENESS IN OLDER ADULTS}

COVID-19 vaccine trials should demonstrate proof of useful vaccine efficacy in terms of protection against severe disease. They should also evaluate the duration of protection by providing con- 
tinued blinded follow-up of the vaccine and placebo groups, even if there is evidence of short-term efficacy. The WHO recommends that vaccines show an estimated risk reduction of at least $50 \%$, with sufficient precision to conclude that the true vaccine efficacy is above $30 \%$. This is because a vaccine with $50 \%$ efficacy may sufficiently reduce the incidence of COVID-19 in those who are vaccinated, although the efficacy far above $50 \%$ is preferable.

In older adults, immune senescence results in an increased vulnerability to respiratory diseases, such as influenza, with a corresponding reduction in vaccine effectiveness. Medical illness, mental and psychosocial health issues, frailty, and functional dependence accelerate changes associated with immune senescence. Combined with reduced adaptive immune response, inflammaging (chronic elevation of inflammatory cytokine levels), and under-regulation of cytokine production, older people tend to have poorer responses to vaccination. Thus, the goal of vaccination in older people is to provide some clinical protection against the disease rather than inducing sterilizing immunity against infections. ${ }^{5)}$

When older people are administered vaccines, antibody responses are commonly evaluated based on the rates of seroprotection (titers above 1:40 by hemagglutination inhibition testing) and seroconversion (at least a four-fold increase in antibody titer compared to that at baseline). ${ }^{6}$

In influenza vaccine studies, antibody responses in older adults were less compared with those observed in younger adults and were not the best correlates for immune protection against infections. Measures of cell-mediated immune responses, such as the ratio of interferon- $\gamma$ to interleukin-10 and the level of cytolytic mediatory granzyme $\mathrm{B}$, were better correlated with protection. This makes sense as cell-mediated immunity plays an important role in preventing respiratory virus infections. ${ }^{7)}$

These findings have several important implications. First, the evaluation of vaccine efficacy in older people through immunological surrogates should measure both antibody and T-cell responses to assess the potential for clinical protection. Moreover, vaccine development for older people should consider novel approaches for antigen presentation, particularly protein content that stimulates cell-mediated immunity, alternative routes of vaccine delivery for an augmented vaccine response, use of higher doses, and use of attenuated-live viruses or virus-like particles. Adjuvants, or substances that enhance both humoral and T-cell-mediated responses via enhanced antigen presentation, activation and maturation of dendritic cells, and production of inflammatory cytokines, may also be added to improve immunogenicity. ${ }^{8)}$

A review of influenza vaccines in older people found that enhanced vaccines using high doses, adjuvants, and intradermal administration resulted in $82 \%, 52 \%$, and $32 \%$ greater titers than standard-dose vaccines. ${ }^{9)}$ While adjuvants improved vaccine immunogenicity, a systematic review and meta-analysis of adjuvanted vaccines also showed higher rates of solicited adverse events. However, these were mostly mild and transient, without causing significant safety concerns. ${ }^{10)}$

Overall, owing to changes in the immune system with age, trials evaluating the response of vaccines in older people should be assessed separately. Immunosenescence leads to defects in innate and adaptive immune responses; thus, vaccine responses tend to be weaker and decline earlier. Therefore, improved vaccination strategies, adjuvants, and vaccines that specifically target the aged immune system may be required. ${ }^{11)}$

\section{TYPES OF COVID-19 VACCINE PLATFORMS}

Several platforms are being explored for vaccines against SARS$\mathrm{CoV}-2$. The four main platforms are virus vaccines, nucleic acid vaccines, viral vector vaccines, and protein-based vaccines. ${ }^{12,13)}$ For inactivated viral vaccines, the viruses should be completely inactivated to avoid causing disease. However, there is an associated risk of vaccine-associated enhanced disease, in which vaccinated people develop more severe disease if they encounter an infection. Nucleic acid vaccines are based on DNA and RNA segments integrated into a plasmid, which when taken up by host cells, allow the virus protein to be manufactured within the cells to mount an immune response. DNA vaccines have a theoretical risk of integrating with host cell DNA, although this has not been observed in animal or human studies. This is less likely with mRNA vaccines, which do not include retrovirus elements for reverse transcription into DNA. $^{14)}$

Viral vector-based vaccines use viral vectors, such as adenoviruses, to introduce genetic sequence coding for the antigen into host cells. However, there is a risk of loss of genetic material coding for the antigen during manufacturing processes, which may result in vaccine failure. Viral proteins produced using recombinant approaches are available but tend to require adjuvants to develop an acceptable immune response. An intermediate between protein subunits and inactivated viruses can be achieved by assembling proteins into virus-like particles (VLPs), which can mimic the wild virus structure but are not infectious owing to the lack of genetic material. The "molecular clamp" enables viral proteins to retain their shape; thus, VLPs can induce a strong immune response. ${ }^{12,13)}$ The route of vaccination is also an important consideration. While intramuscular injections can lead to protective IgG antibodies in the respiratory mucosa, they are less effective in inducing mucosal IgA antibodies or T-cell responses in the lungs. The respiratory mucosal route is more adapted to this function, as well as generat- 
ing macrophage-mediated immunity. ${ }^{15)}$

Inactivated virus, protein subunit, and nucleic acid vaccines cannot be administered via the respiratory mucosa as this mode of administration may not be safe with immune adjuvants and repeated delivery. However, recombinant viral-vectored vaccines, particularly those using human serotype 5 adenovirus (Ad5) or chimpanzee-derived adenovirus (ChAd), have been shown to be safe and effective for respiratory mucosal vaccination with other vaccines. ${ }^{16)}$ Thus, to develop safe and effective COVID-19 vaccines, the vaccine platform, adjuvant, excipient, dose, and route of administration must be considered. ${ }^{17)}$

Table 1 summarizes the candidate vaccines and vaccine platforms that are already in phase $2 / 3$ or phase 3 trials (as of January $1,2021)$, along with the age groups of participants to be enrolled.

\section{EVIDENCE FROM COVID-19 VACCINE TRIALS RELEVANT TO OLDER ADULTS}

Evaluation of the results of COVID-19 vaccine trials relevant to older adults requires a review of immunogenicity, i.e., the ability of vaccines to induce an immune response, and reactogenicity, i.e., the likelihood of adverse events owing to the vaccine.

The two mRNA vaccines, BNT162b2 from Pfizer-BioNTech and mRNA-1273 by Moderna, were the first COVID-19 vaccines to demonstrate efficacy in phase 3 trials. ${ }^{18,19)}$ In the BNT162b2 phase 3 study, 43,548 participants were randomized to receive two $30-\mu \mathrm{g}$ doses of the vaccine or saline placebo 21 days apart. The study enrolled people aged 16 years and older, with $42 \%$ of participants aged above 55 years. The vaccine showed $95 \%$ efficacy in protecting against SARS-CoV-2 infections, with eight cases occurring in the BNT162b2 group and 162 cases in the placebo group. In terms of reactogenicity, the most common adverse events were mild to moderate pain at the injection site, fatigue, and headache. These adverse events were transient, with a low incidence of serious adverse events. When the two age groups (16-55 and > 55 years) were compared, the second dose was associated with more local and systemic adverse events, while there was less reactogenicity in the older age group. ${ }^{18)}$

In the phase 3 trial of the mRNA-1273 vaccine, 30,420 participants aged 18 years and older were randomized to receive two 100 $\mu \mathrm{g}$ doses of mRNA-1273 or saline placebo 28 days apart. The vaccine showed an efficacy of $94.1 \%$, with 11 cases occurring in the mRNA-1273 vaccine group versus 185 cases in the placebo group. The rates of adverse events in the vaccine and placebo groups were $84.2 \%$ and $19.8 \%$, respectively, for the first dose and $88.6 \%$ and $18.8 \%$, respectively, for the second dose. While reactogenicity increased from the first to the second dose, the incidence of serious adverse events was low. Similar to the BNT162b2 vaccine, the rates of solicited injection site and systemic adverse events were higher in the younger (18-65 years) group than in the older (65 years and above) participants. ${ }^{19)}$

For these mRNA vaccines, the higher rate of immunogenicity likely translated into the high efficacy rate in reducing SARS$\mathrm{CoV}-2$ infection, as seen in the phase 3 studies. While there are theoretical concerns regarding reduced immunogenicity with age, phase 1 dose-escalation trials of the mRNA-1273 vaccine observed similar effective antibody responses in three age groups (18-55, 56-70, and 71 years and older). The antibody titers measured at three different time points (summarized in Table 2) suggested that mRNA vaccines are likely to be effective in older people. ${ }^{20,21)}$

The other vaccine with published phase 3 results is ChAdOx1 nCoV-19 (AZD1222), an adenovirus-vectored vaccine by AstraZeneca, with interim results available showing its safety and efficacy in 11,636 participants. AZD1222 was compared to a control vaccine, in which two doses were administered 28 days apart. Among participants receiving two standard doses of AZD1222, the efficacy was $62.1 \%$ (27 cases in AZD1222, 71 cases in placebo). However, a subgroup of participants inadvertently administered a lower dose of the vaccine for the first injection showed a 90.0\% efficacy rate ( 3 cases in AZD1222; 30 cases in placebo). ${ }^{22)}$ Unfortunately, the results from the different dosing regimens could not be compared owing to effects from multiple confounding factors, particularly the dosing interval. A longer dosing interval between both doses may result in better immunogenicity, which was generally longer in the low-dose group. ${ }^{23)}$ This uncertainty regarding the optimal dose for AZD1222 requires further study before any firm conclusions can be made.

In terms of safety data, the interim results contained data from 74,341 person-months of follow-up. There were 175 severe adverse events, of which 84 occurred with AZD1222 and 91 in the control group. ${ }^{22)}$ A case of transverse myelitis (an idiopathic short-segment spinal cord demyelination) was reported as possibly related to the vaccine. Two other cases of transverse myelitis also occurred during follow-up, which were unrelated to AZD1222; one was deemed unlikely to be vaccine-related as the patient had previously undiagnosed multiple sclerosis and the other patient had received the MenACWY vaccine. Generally, AZD1222 showed a good safety profile, with similar rates of adverse events across the study arms. The phase 2/3 trial of AZD1222 also demonstrated lower rates of solicited local and systemic adverse events in the older age group and after the second vaccine dose. ${ }^{24)}$

Finally, the Ad26.COV2.S vaccine is an adenovirus serotype 26 (Ad26) vector by Janssen Pharmaceuticals. This vaccine deserves mention as it is planned as a single-dose vaccine, which is likely to 


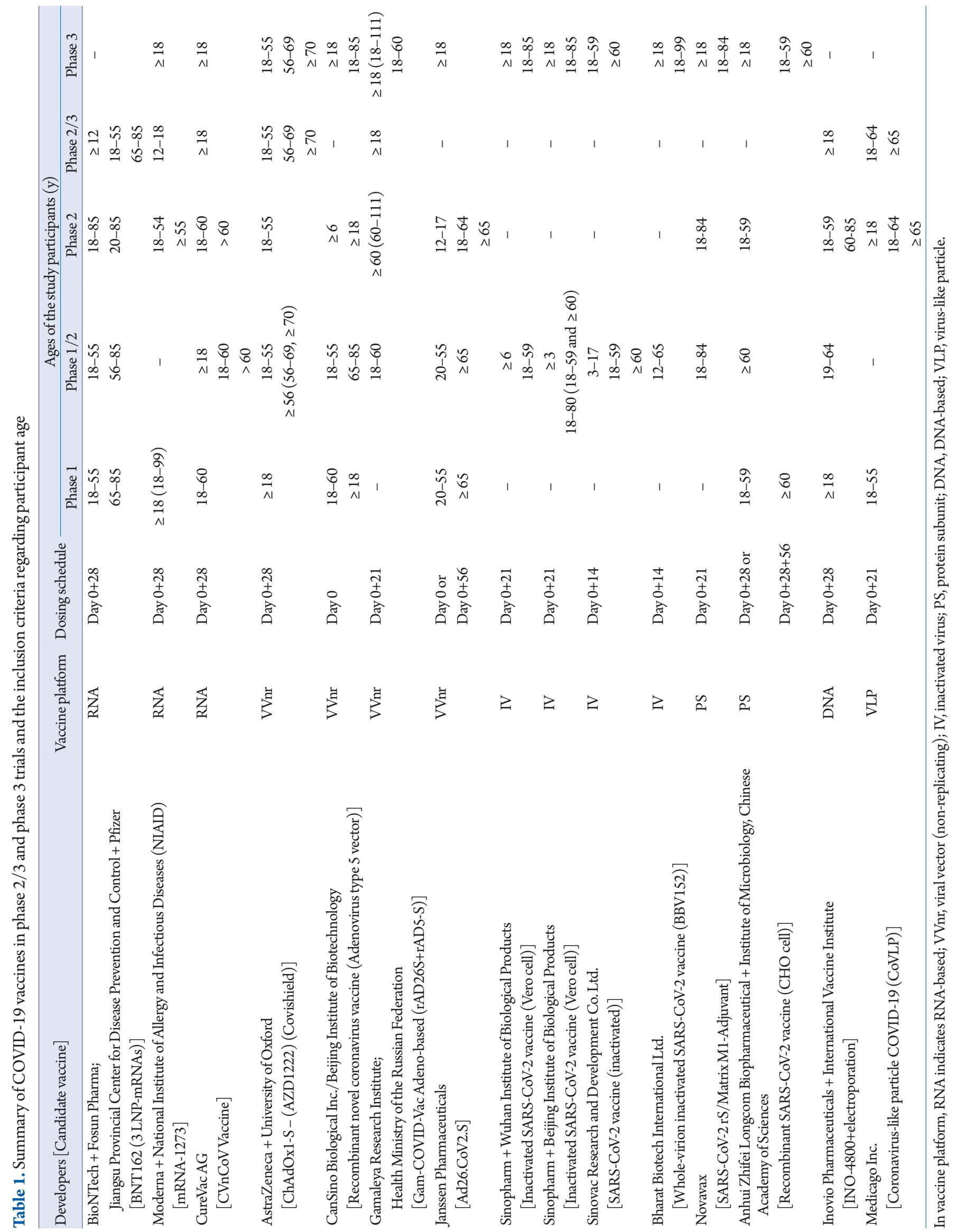


Table 2. ELISA anti-S2P endpoint titers at 28 days post-vaccinations with mRNA-1273 and 90 days after the second vaccination ${ }^{20,21)}$

\begin{tabular}{|c|c|c|c|c|c|c|c|}
\hline \multirow{2}{*}{ Age group (y) } & \multirow{2}{*}{ Dose $(\mu \mathrm{g})$} & \multicolumn{2}{|c|}{ Day 29} & \multicolumn{2}{|c|}{ Day 57} & \multicolumn{2}{|c|}{ Day 119} \\
\hline & & GMTs & $95 \% \mathrm{CI}$ & GMTs & $95 \% \mathrm{CI}$ & GMTs & $95 \% \mathrm{CI}$ \\
\hline \multirow[t]{3}{*}{$18-55$} & 25 & 40,227 & $18,723-55,587$ & 299,751 & $206,071-436,020$ & - & - \\
\hline & 100 & 109,209 & $79,050-150,874$ & 782,719 & $619,310-989,244$ & 235,228 & $117,236-312,195$ \\
\hline & 250 & 213,526 & $128,832-353,896$ & $1,192,154$ & $924,878-1,536,669$ & - & - \\
\hline \multirow[t]{3}{*}{$56-70$} & 25 & 17,684 & $5,300-59,001$ & 323,945 & $182,202-575,958$ & & \\
\hline & 100 & 115,831 & $73,288-183,069$ & $1,183,066$ & $379,698-3,686,201$ & 151,761 & $88,571-260,033$ \\
\hline & 250 & - & - & - & - & - & - \\
\hline \multirow[t]{3}{*}{$\geq 71$} & 25 & 57,986 & $31,452-106,905$ & $1,128,391$ & $636,087-2,001,717$ & - & - \\
\hline & 100 & 203,365 & $97,384-424,686$ & $3,638,522$ & $1,316,233-10,058,130$ & 157,946 & $94,345-264,420$ \\
\hline & 250 & - & - & - & - & - & - \\
\hline
\end{tabular}

Comparisons between different age groups of T-cell responses are not shown here as interpretation is more complex. The preferred response would be a bias toward the expression of Th1 cytokines (tumor necrosis factor $\alpha>$ interleukin- $2>$ interferon- $\gamma$ ), with minimal Th2 cytokine expression (interleukins 4 and 13 ). Surrogate measures of immunogenicity such as SARS-CoV-2-binding antibody responses, neutralizing responses, and T-cell responses are indicative only of clinical efficacy, which requires confirmation in phase 3 trials.

Antibody titers may not be comparable among trials because of the use of different assays; thus, comparisons are usually made to convalescent serum levels. ELISA, enzyme-linked immunosorbent assay; S2P, site-2 protease; GMTs, geometric mean titers.

improve compliance, particularly for older people. In the phase 1-2a trial, 805 participants aged $18-55$ and 65 years or older were randomized to receive Ad26.COV2.S at a dose of $5 \times 10^{10}$ viral particles (low-dose) or $1 \times 10^{11}$ viral particles (high-dose) per milliliter or placebo (as one or two-dose regimens). A single dose of Ad26.COV2.S resulted in a strong humoral response independent of the age group or vaccine dose. These titers further increased and stabilized at 71 days follow-up after the first dose, suggesting a durability of response after a single dose. The most frequent systemic adverse events were fever, fatigue, headache, myalgia, and injection site pain. There was less reactogenicity in the older age group for the low dose and after a second dose. ${ }^{25}$

\section{CONCLUSION}

Older people are vulnerable to developing SARS-CoV-2 infections and complications; thus, they are a priority group for preventive vaccination campaigns. A general understanding of the immune response of older people to vaccines and the various COVID-19 vaccine platforms is required, in addition to current knowledge of evidence from ongoing available phase 3 trials of vaccines specific to older people to evaluate the risks and benefits of different vaccines for this population.

\section{ACKNOWLEDGMENTS}

\section{CONFLICT OF INTEREST}

The researcher claims no conflicts of interest.

\section{FUNDING}

None.

\section{REFERENCES}

1. World Health Organization. The COVID-19 candidate vaccine landscape [Internet]. Geneva, Switzerland: World Health Organization; 2021 [cited $2021 \mathrm{Feb} 11$ ]. Available from: https:// www.who.int/publications/m/item/draft-landscape-of-covid19-candidate-vaccines.

2. US Food and Drug Administration. COVID-19 vaccines [Internet]. Silver Spring, MD: Food and Drug Administration; 2021 [cited 2021 Feb 11]. Available from: https://www.fda.gov/ emergency-preparedness-and-response/coronavirus-disease-2019-covid-19/covid-19-vaccines.

3. World Health Organization. WHO SAGE Roadmap for prioritizing uses of COVID-19 vaccines in the context of limited supply [Internet]. Geneva, Switzerland: World Health Organization; 2020 [cited 2021 Feb 11]. Available from: https://www. who.int/docs/default-source/immunization/sage/covid/ sage-prioritization-roadmap-covid19-vaccines.pdf?sfvrsn $=\mathrm{b}$ f227443_36\&download $=$ true.

4. Krause P, Fleming TR, Longini I, Henao-Restrepo AM; Peto R; World Health Organization Solidarity Vaccines Trial Expert Group. COVID-19 vaccine trials should seek worthwhile efficacy. Lancet 2020;396:741-3.

5. McElhaney JE, Kuchel GA, Zhou X, Swain SL, Haynes L. T-cell immunity to influenza in older adults: a pathophysiological framework for development of more effective vaccines. Front Immunol 2016;7:41. 
6. Deans GD, Stiver HG, McElhaney JE. Influenza vaccines provide diminished protection but are cost-saving in older adults. J Intern Med 2010;267:220-7.

7. McElhaney JE, Xie D, Hager WD, Barry MB, Wang Y, Kleppinger A, et al. T cell responses are better correlates of vaccine protection in the elderly. J Immunol 2006;176:6333-9.

8. McElhaney JE, Coler RN, Baldwin SL. Immunologic correlates of protection and potential role for adjuvants to improve influenza vaccines in older adults. Expert Rev Vaccines 2013;12:75966.

9. Ng TW, Cowling BJ, Gao HZ, Thompson MG. Comparative immunogenicity of enhanced seasonal influenza vaccines in older adults: a systematic review and meta-analysis. J Infect Dis 2019;219:1525-35.

10. Baay M, Bollaerts K, Verstraeten T. A systematic review and meta-analysis on the safety of newly adjuvanted vaccines among older adults. Vaccine 2018;36:4207-14.

11. Weinberger B, Herndler-Brandstetter D, Schwanninger A, Weiskopf D, Grubeck-Loebenstein B. Biology of immune responses to vaccines in elderly persons. Clin Infect Dis 2008;46:1078-84.

12. World Health Organization. COVID-19 vaccines: safety surveillance manual: description and general safety considerations for implementation [Internet]. Geneva, Switzerland: World Health Organization; 2020 [cited $2021 \mathrm{Feb} 11$ ]. Available from: https://www.who.int/vaccine_safety/committee/Module Vaccine_description.pdf?ua $=1$.

13. Haque A, Pant AB. Efforts at COVID-19 vaccine development: challenges and successes. Vaccines (Basel) 2020;8:739.

14. Liu MA. A comparison of plasmid DNA and $m R N A$ as vaccine technologies. Vaccines (Basel) 2019;7:37.

15. Jeyanathan M, Afkhami S, Smaill F, Miller MS, Lichty BD, Xing Z. Immunological considerations for COVID-19 vaccine strategies. Nat Rev Immunol 2020;20:615-32.

16. Afkhami S, Yao Y, Xing Z. Methods and clinical development of adenovirus-vectored vaccines against mucosal pathogens. Mol Ther Methods Clin Dev 2016;3:16030.

17. Wang J, Peng Y, Xu H, Cui Z, Williams RO. The COVID-19 vac- cine race: challenges and opportunities in vaccine formulation. AAPS PharmSciTech 2020;21:225.

18. Polack FP, Thomas SJ, Kitchin N, Absalon J, Gurtman A, Lockhart S, et al. Safety and efficacy of the BNT162b2 mRNA Covid-19 vaccine. N Engl J Med 2020;383:2603-15.

19. Baden LR, El Sahly HM, Essink B, Kotloff K, Frey S, Novak R, et al. Efficacy and safety of the mRNA-1273 SARS-CoV-2 vaccine. N Engl J Med 2021;384:403-16.

20. Jackson LA, Anderson EJ, Rouphael NG, Roberts PC, Makhene $\mathrm{M}$, Coler RN, et al. An mRNA vaccine against SARS-CoV-2: preliminary report. N Engl J Med 2020;383:1920-31.

21. Anderson EJ, Rouphael NG, Widge AT, Jackson LA, Roberts PC, Makhene M, et al. Safety and immunogenicity of SARSCoV-2 mRNA-1273 vaccine in older adults. N Engl J Med 2020;383:2427-38.

22. Voysey M, Clemens SA, Madhi SA, Weckx LY, Folegatti PM, Aley PK, et al. Safety and efficacy of the ChAdOxl nCoV-19 vaccine (AZD1222) against SARS-CoV-2: an interim analysis of four randomised controlled trials in Brazil, South Africa, and the UK. Lancet 2021;397:99-111.

23. Medicines and Healthcare products Regulatory Agency. COVID-19 vaccine AstraZeneca, solution for injection in multidose container: COVID-19 vaccine (ChAdOx1-S [recombinant]) [Internet]. London, UK: Medicines and Healthcare products Regulatory Agency; 2021 [cited 2021 Feb 11]. Available from: https://assets.publishing.service.gov.uk/government/uploads/system/uploads/attachment_data/file/949772/UKPAR COVID_19_Vaccine_AstraZeneca_05.01.2021.pdf.

24. Ramasamy MN, Minassian AM, Ewer KJ, Flaxman AL, Folegatti PM, Owens DR, et al. Safety and immunogenicity of ChAdOx1 $\mathrm{nCoV}-19$ vaccine administered in a prime-boost regimen in young and old adults (COV002): a single-blind, randomised, controlled, phase 2/3 trial. Lancet 2021;396:1979-93.

25. Sadoff J, Le Gars M, Shukarev G, Heerwegh D, Truyers C, de Groot AM, et al. Interim results of a phase 1-2a trial of Ad26. COV2.S Covid-19 vaccine. N Engl J Med 2021 Jan 13 [Epub]. http://doi.org/10.1056/NEJMoa2034201. 